\title{
A response to the Chief Medical Officer's report on Genomic Medicine: a catalyst for transformation
}

\author{
Julian Barwell*,1,2, Jacqui Shaw², Ming Lim³ , Riddhi Y Shukla ${ }^{2}$ \& Joanna Lowry ${ }^{1}$ \\ ${ }^{1}$ University Hospitals of Leicester, Leicester Royal Infirmary, Leicester LE1 5WW, UK \\ ${ }^{2}$ University of Leicester, Department of Cancer Studies \& Molecular Medicine, Leicester LE1 7RH, UK \\ ${ }^{3}$ University of Liverpool, Management School, Foundation Building, Brownlow Hill, Liverpool L69 7ZX, UK \\ * Author for correspondence: julian.barwell@uhl-tr.nhs.uk
}

"Dame Sally's vision is extremely welcome as it focuses minds on the need for dynamic leadership around transformation."

First draft submitted: 4 August 2017; Accepted for publication: 7 September 2017; Published online: 21 December 2017

Keywords: biobanks • bioinformatics • genetics • genomics $\bullet$ Leicester $\bullet$ molecular medicine $\bullet$ personalized medicine $\bullet$ policy issues $\bullet$ transformation

On the 4 July 2017, the chief medical officer for England, DS Davies, set out a vision for genomic medicine over the next 5 years [1]. Her vision is that genomic testing should become a normal part of the National Health Service (NHS) care, ushering in the era of precision medicine, the "targeting of drugs to do the most good and least harm" beginning with cancer patients and those with rare diseases. This was based on the fact that whole genome testing is falling in cost to under US\$1000 per genome [2] but the complexity of interpreting the data obtained requires a step change with new investment in bioinformatics and large collaborations on databasing genomic variants. The establishment of Genomics England and the 100,000 Genomes Project has placed us ahead of the game and is a catalyst for innovation [3].

DS Davies made three key points which bring together the concept of standardization and consolidation. First, that local genetic laboratories are currently akin to cottage industries and are ill-equipped for the expected changes in scale. Second, that patients should have the right to expect personalized medicine when it comes to rare diseases and in particular cancer, where six out of ten patients will receive information from whole genome sequencing of blood and/or tumor that can help target treatment [4]. Finally, new targeted genetic tests for specific personalized medicine indications should be developed. Dame Sally used identifying predicted antibiotic resistance in tuberculosis [5], which is established in Leicester, as an example of good practice.

This approach of targeting new genetic tests could also be applied to pharmacogenetics, predicting cancer risk in population cohorts, noninvasive genetic testing in pregnancy and cell-free circulating tumor DNA in plasma to detect temporal changes in patients with cancer as a test for disease monitoring [6].

This targeted molecular medicine technology and genomic testing lead to four aims:

- Treat disease for what it is rather than what it looks like down a microscope

- Treat people with the right drug at the right dose the first time

- Screen based on risk rather than age alone

- Move to a world beyond microbiology cultures and microscopes

Under the umbrella of standardization and consolidation, there is an expectation that the indications for, and access to, genetic and genomic testing will be applied uniformly throughout the UK. These tests will be agreed nationally by leading clinicians and academics with economic modeling and then carried out in a standard fashion in a small number of supra-regional laboratories.

This exciting vision has five challenges: 
- The culture of change around healthcare systems is based on protectionism, cynicism about the benefit of genetics in everyday practice, a lack of education and understanding of the implications and interpretation and concerns about confidentiality;

- Implementation of parallel working streams around clinical transformation and financial planning (including building adequate bioinformatics capacity) with such service development occurring alongside current processes and service delivery;

- The IT and staff training requirements for multidisciplinary meetings and manipulation of large datasets. The collation, storage and analysis of these germline and somatic datasets at diagnosis and subsequent follow-up visits have enormous therapeutic decision making potential but have considerable technological and man-power implications which require integrated economic modeling;

- The linking of diagnostic and therapeutic commissioning on a national level;

- Appropriate investment in technology and education to develop new services.

Overcoming these barriers requires considerable buy-in from key stakeholders, commissioners and hospital management as well as national investment schemes supported by a clear political vision and integrated transformational institutions such as the Clinical Research Network, the Academic Health Science Networks and the Collaborative Leadership in Applied Health Research and Care partnerships.

\section{What is the likely impact of this vision on clinical genetics \& local laboratory services \& how should it prepare for the future?}

As the number and availability of tests and their complexity increases, the role of the clinical geneticist is likely to change from a gatekeeper of testing and diagnostician to a leader of transformation, result interpretation, cascading and designing clinical care models.

The training of clinical geneticists classically involves a focus on calculating the likelihood of identifying Mendelian disease through genetic testing and describing/recognizing dysmorphological features and syndromic patterns recognition. These are under-pinned by family history taking and discussions on individual patient's potential coping mechanisms around result giving that may predict future personal and familial risk.

Falling costs and improved actionability around genetic testing mean that the importance of family history on predicting mutation detection rates, gatekeeping access to genetic testing based on risk; using dysmorphology to decide on targeted genetic testing; the need for genetic counseling around diagnostic testing to understand the cause of and predict the course of disease are all being challenged.

There is a growing emphasis on working with treating physicians around the interpretation of complex genomic data and understanding the significance of variants to determine if the variant detected helps explain the observed clinical picture, which is a type of 'reverse-phenotyping', in other words, the genetic test will be carried out before history and examination. This will involve significant increase in the use of database entries and searches. The problem is that transformative leadership and education around collaborative working, bioinformatics, database entry and searches are not classically funded in the same way as direct clinical contact with referred patients. For example, we have been involved in over 70 initiatives around genomic medicine from developing clinical research facilities to introducing genomic medicine eligibility criteria aids for clinical to collaborative patient and public initiatives. These roles and the benefit of novel diagnostic testing techniques on developing more cost-effective targeted treatments and screening will need economic modeling. Classic departmental commissioning does not take into consideration personalized medicine where diagnostics improve targeted therapeutic decision making.

Some things will remain unchanged in clinical genetics including family planning, communicating risk and inheritance patterns through cascading of results to relatives, managing family dynamics and exploring coping mechanisms around perceived future risk. A key question is whether clinical geneticists will embrace clinical care modeling and delivery for complex multisystem disease in a similar way as they have done for von Hippel-Lindau syndrome and Neurofibromatosis type 2 . This is particularly relevant for newly discovered conditions where the phenotype and disease course is not well known and requires further assessment. Primary care colleagues may find these conditions difficult to manage and require expert guidance. It is clear that the planning, commissioning and delivery of testing, interpretation and care in the genomic era require further consideration.

In the light of all these factors, the role of genetics counselors, family members, systems of consent, privacy, confidentiality, data protection and the role of the press and media in communicating a coherent, yet accurate, 
picture of the implications of novel diagnostic techniques will all have wide ranging and profound effects upon patient choice and decision making.

Furthermore, clinical transformation is widely understood as extremely challenging to implement due to the difficulties associated with change management, attitude shifts and parallel busy workloads. For example, established clinicians that have not heard of, been trained on or used new technologies may not be prepared to appreciate or accept the potential relevance of change and its benefit to healthcare. "I care passionately for my patients and have dedicated my working life to them. Therefore I understand clinical reality in every day practice rather than research institutes and know what is best for them". This position is understandable when you consider that clinicians are often very busy trying to deliver equitable and high-quality care. This long-term pressure leaves proud and high-achievers feeling unable both from a time and financial perspective to invest in change. Perhaps, this is compounded by a subconscious fear of the unknown and the potential impact on their role.

The commercial sector however sees a growing interest in the public's and patients' desire to access new technologies from a social personal understanding of heritage and potential impact on health. The view that the individual is an ever-changing data repository waiting to be unlocked with the relevant tests is more and more common, leading to public empowerment choices which may impact on therapeutic choices and prognostic outcomes. This translates into a growing commercial market opportunity with the global genetic testing market poised to reach approximately US $\$ 10.3$ billion by 2025 [7], enabling many commercial players to position themselves in this field to take advantage of this lucrative market. Concerns regarding validation of results to international clinical laboratory standards, informed consent around the interpretation of results and their applicability for the worried well have not impacted on the appetite for these services and may, in time, impact on or compete with clinical services.

\section{So how can clinicians find partnerships to lever change?}

Universities and the commercial sector can certainly help. Universities have four main roles in developing genomic capacity:

- Key staff providing leadership and expertise for transformational change; developing a consortium to drive and oversee change;

- Developing and offering relevant undergraduate and postgraduate courses;

- Providing networking opportunities for industry collaborations;

- In partnership with transformational partners, developing new clinical services from bench to bedside.

The commercial and international sector is also keen to develop links to improve access to drugs and increase accessibility to market. As NHS funds for innovation are limited, mutually beneficial arrangements around preferred partners for personalized medicine service development initiatives will increase efficiency. These will be based on local expertise and build confidence around investment if market share is perceived to be greater in larger consortia. Clinically, this also has advantages as larger and more accessible genomic databases will improve our understanding of human variation $[8]$.

Dame Sally's vision is extremely welcome as it focuses minds on the need for dynamic leadership around transformation. This involves reconfiguration of local molecular and cytogenetic services to a split model of larger supraregional centers offering gene panels and whole genome sequencing alongside a fundamental re-think of the way local histopathology, malignant haematology, microbiology and pharmacology services are designed. Although it currently offers no new money, it is incredibly helpful to local innovators keen to influence and work with the public, patients, the commercial sector, clinicians, scientists, hospital boards and commissioners to make this a reality.

Financial \& competing interests disclosure

The authors have no relevant affiliations or financial involvement with any organization or entity with a financial interest in or financial conflict with the subject matter or materials discussed in the manuscript. This includes employment, consultancies, honoraria, stock ownership or options, expert testimony, grants or patents received or pending, or royalties. Dr Julian Barwell is also employed by the East Midlands Clinical Research Network and has received honoraria from Astra Zeneca and Novartis for work unrelated to this article.

No writing assistance was utilized in the production of this manuscript. 


\section{References}

1 Davies S C Prof Dame. Generation genome (2016). www.gov.uk/government/publications/chief-medical-officer-annual-report-2016-generation-genome

2 National Human Genome Research Institute. www.genome.gov/27565109/the-cost-of-sequencing-a-human-genome/

3 Genomics England. www.genomicsengland.co.uk/about-genomics-england/

4 Akhmetov I, Bubnov RV. Assessing value of innovative molecular diagnostic tests in the concept of predictive, preventive, and personalized medicine. EPMA J. 6, 19 (2015)

5 Pérez-Osorio AC, Boyle DS, Ingham ZK et al. Rapid identification of mycobacteria and drug-resistant mycobacterium tuberculosis by use of a single multiplex PCR and DNA sequencing. J. Clin. Microbiol. 50(2), 326-336 (2012).

6 Shaw JA, Brown J, Coombes RC et al. Circulating tumor cells and plasma DNA analysis in patients with indeterminate early or metastatic breast cancer. Biomark. Med. 5(1), 87-91 (2011).

7 Global Personalized Medicine Market Analysis \& Trends - Industry Forecast to 2025. Accuray Research (2017). www.accurayresearch.com/personalized-medicine-market-market-analysis-size-share-trends

8 Frazer KA, Murray SS, Schork N Nicholas J, Topol EJ. Human genetic variation and its contribution to complex traits. Nat. Rev. Genet. 10(4), 241-251 (2009) 